\title{
Valutazione endocrinologica del paziente con ginecomastia
}

\author{
Laura Rossi $^{1} \cdot$ Pietro Locantore $^{1} \cdot$ Alfredo Pontecorvi $^{1}$
}

Accettato: 26 gennaio 2021 / Pubblicato online: 31 maggio 2021

(c) The Author(s) 2021

\section{Introduzione}

La ginecomastia è una condizione caratterizzata dalla proliferazione della ghiandola mammaria nel soggetto di sesso maschile.

Solitamente è una condizione benigna, tuttavia nello $0,2 \%$ dei casi si associa a carcinoma mammario del maschio. È generalmente bilaterale, sebbene nel $40 \%$ dei casi può insorgere monolateralmente (soprattutto nelle fasi iniziali).

È una condizione riscontrabile in tutte le fasce di età con eziologie diverse, in particolare nel periodo neonatale, in fase peripuberale e nell'età adulta compresa fra 50 e 70 anni.

La ginecomastia vera va differenziata dalla pseudoginecomastia (ginecomastia falsa), definita anche adipomastia, per la proliferazione del tessuto adiposo e non dello struma ghiandolare mammario, spesso presente nelle condizioni di sovrappeso/obesità [1].

\section{Eziopatogenesi}

Le cause di ginecomastia vera sono molteplici ma trovano una condizione di base comune: un alterato rapporto androgeni/estrogeni (fisiologicamente pari a 3).

$\mathrm{Nel}$ neonato è una condizione transitoria che si risolve spontaneamente in poche settimane e nasce dall' azione degli estrogeni di origine placentare che permangono in circolo.

Nella fase peripuberale, in conseguenza all'aumento fisiologico delle gonadotropine, si verifica l'aumento di estrogeni, a cui si associa l'incremento dei livelli di SHBG e di

P. Locantore

pietro.locantore@icloud.com

1 Dipartimento di Endocrinologia e Diabetologia, Fondazione Policlinico Universitario Agostino Gemelli IRCCS, Università Cattolica del Sacro Cuore, Roma, Italia

androgeni surrenalici che vengono aromatizzati in estrogeni. Anche questa condizione è per lo più transitoria e si risolve spontaneamente.

La causa iatrogena è fra le più frequenti, soprattutto nell'età adulta, e consegue all'assunzione di farmaci che interferiscono con gli ormoni sessuali (Tab. 1).

In presenza di ginecomastia è necessario escludere cause neoplastiche.

La causa più frequente è il prolattinoma (la prolattina non ha un ruolo diretto nella stimolazione della ghiandola mammaria ma coadiuva l'azione degli estrogeni e inibisce la secrezione pulsatile del GnRH ipotalamico). Inoltre, è necessario considerare tumori testicolari o non testicolari secernenti HCG (come i germinomi, neoplasie gastrointestinali, ecc.), tumori surrenalici secernenti Delta4-androstenedione, convertito in estrogeni, oppure tumori ipofisari secernenti gonadotropine.

Bisogna anche considerare l'eventuale presenza di ipogonadismo primitivo e secondario, tra cui si annovera la sindrome di Klinefelter, poiché tali soggetti hanno un rischio di sviluppo di carcinoma mammario molto più elevato rispetto alla popolazione generale.

Tra le cause di ginecomastia si annovera anche l'ipertiroidismo e la cirrosi epatica, che inducono aumento periferico della conversione, e l'insufficienza renale cronica.

$\mathrm{Nel} 25 \%$ dei casi, la ginecomastia risulta in assenza di cause specifiche [2].

\section{Diagnosi}

La diagnosi parte dall'esame obiettivo da eseguire in posizione supina. Il grado di sviluppo della mammella è classificato secondo la classificazione di Tanner. 
Tabella 1 Farmaci che interferiscono con gli ormoni sessuali

\begin{tabular}{ll}
\hline Meccanismo d'azione & Farmaco \\
\hline $\begin{array}{l}\text { azione estrogeno-mimetica } \\
\text { stimolanti la produzione di PRL }\end{array}$ & $\begin{array}{l}\text { digitale, fitoestrogeni, fenotiazine, reserpina, marijuana } \\
\text { antistaminici, antidepressivi triciclici, alfa-metildopa, inibitori selettivi del reuptake della serotonina, } \\
\text { butirrofenoni, fenitoina }\end{array}$ \\
$\begin{array}{l}\text { interferenti con il recettore del } \\
\text { sestosterone }\end{array}$ & $\begin{array}{l}\text { analoghi dell'LH-RH, ketoconazolo, spironolattone, eroina, citotossici } \\
\text { riducono la sintesi di androgeni } \\
\text { ignoto }\end{array}$ \\
\hline
\end{tabular}

Se si sospetta un'interferenza farmacologica, è utile sospendere il farmaco rivalutare il paziente a distanza. È opportuno eseguire il dosaggio di FSH, LH, testosterone totale, PRL, estradiolo, beta-HCG, TSH; è utile controllare la funzione epatica e renale. L'ecografia mammaria è utile per distinguere fra ginecomastia vera e falsa. In caso di dubbi o sospetti neoplastici, possono essere eseguite indagini radiologiche di secondo livello (mammografia, RMN mammaria) [3].

\section{Terapia}

I pazienti con forme transitorie solitamente non vengono trattati poiché sono autorisolventi, a meno che non vi sia un alto disagio psicologico da parte del paziente. Se la ginecomastia è secondaria a cause neoplastiche, è necessario trattare la neoplasia sottostante. Nel caso di prolattinomi, il quadro migliora dopo il trattamento con dopamino-agonisti. Nel caso di cause farmacologiche, è necessario sospendere il trattamento, se possibile. I pazienti che non rientrano in queste categorie possono essere trattati con antiestrogeni (solitamente il tamoxifene) oppure con inibitori dell'aromatasi (Anastrazolo o Letrozolo). Il trattamento dovrebbe essere iniziato tempestivamente (ginecomastia insorta da meno di 6 mesi e con stadio Tanner 1 o 2) perché la ghiandola mammaria deve essere in attiva fase proliferativa e, solitamente, viene proseguito per 6 mesi con buona risposta. I pazienti affetti da ginecomastia secondaria a ipogonadismo possono avere un miglioramento dopo la somministrazione del testosterone. L'impiego del diidrotestosterone sulla regione mammaria in formulazione gel è, ad oggi, limitato, soprattutto per problematiche di approvvigionamento. L'uso del testosterone nei soggetti con normali livelli di testosterone (quindi con iperestrogenismo relativo) è sconsigliato, poiché vi potrebbe essere un peggioramento del quadro in seguito all'aumento della conversione.

Nei pazienti che iniziano terapie antiandrogeniche per tumori prostatici ormonosensibili si può valutare l'uso della radioterapia locale con 4-6 Gy (anche preventivamente).
Nei casi in cui la ghiandola non sia più in fase proliferativa o in presenza di controindicazioni o fallimenti della terapia medica, la terapia di scelta rimane risulta chirurgica (mastectomia).

Funding Note Open access funding provided by Università Cattolica del Sacro Cuore within the CRUI-CARE Agreement.

Conflitto di interesse Gli autori Pietro Locantore, Laura Rossi e Alfredo Pontecorvi dichiarano di non avere conflitti di interesse.

Consenso informato Lo studio presentato in questo articolo non ha richiesto sperimentazione umana.

Studi sugli animali Gli autori di questo articolo non hanno eseguito studi sugli animali.

Nota della casa editrice Springer Nature rimane neutrale in riguardo alle rivendicazioni giurisdizionali nelle mappe pubblicate e nelle affiliazioni istituzionali.

Open Access This article is licensed under a Creative Commons Attribution 4.0 International License, which permits use, sharing, adaptation, distribution and reproduction in any medium or format, as long as you give appropriate credit to the original author(s) and the source, provide a link to the Creative Commons licence, and indicate if changes were made. The images or other third party material in this article are included in the article's Creative Commons licence, unless indicated otherwise in a credit line to the material. If material is not included in the article's Creative Commons licence and your intended use is not permitted by statutory regulation or exceeds the permitted use, you will need to obtain permission directly from the copyright holder. To view a copy of this licence, visit http://creativecommons.org/licenses/by/4.0/.

\section{Bibliografia}

1. Kanakis GA, Nordkap L, Bang AK et al (2019) EAA clinical practice guidelines-gynecomastia evaluation and management. Andrology 7(6):778-793

2. Narula HS, Carlson HE (2007) Gynecomastia. Endocrinol Metab Clin N Am 36(2):497-519

3. Narula HS, Carlson HE (2014) Gynaecomastia - pathophysiology, diagnosis and treatment. Nat Rev Endocrinol 10(11):684-698 